\title{
Brownian Motion Limit of Random Walks in Symmetric Non-Homogeneous Media
}

\author{
Domingos H. U. Marchetti and Roberto da Silva \\ Instituto de Física \\ Universidade de São Paulo \\ P. O. Box 66 318, 05315-970, São Paulo, SP, Brazil
}

Received 5 April, 1999

\begin{abstract}
The phenomenon of macroscopic homogenization is illustrated with a simple example of diffusion. We examine the conditions under which a $d$-dimensional simple random walk in a symmetric random media converges to a Brownian motion. For $d=1$ Cboth the macroscopic homogeneity condition and the diffusion coefficient can be read from an explicit expression for the Green's function. Except for this case $\mathrm{T}$ the two available formulas for the effective diffusion matrix $\kappa$ do not explicit show how macroscopic homogenization takes place. Using an electrostatic analogy due to Anshelevich $\Gamma$ Khanin and Sinai [AKS] $\Gamma$ we discuss upper and lower bounds on the diffusion coefficient $\kappa$ for $d>1$.
\end{abstract}

\section{Introduction}

The long time behavior of random walks on a random environment is reviewed. We focus mainly on the following question:

What are the conditions under which a properly scaled random walk on a non-homogeneous medium converges to a Brownian motion.

This and related phenomena are usually named macroscopic homogenization (and the environmental conditions are called macroscopic homogeneity conditions) because such system looks homogeneous at macroscopic scales. The discussion will be restricted to simple random walks with non-vanishing transition probabilities (or rates) $\left\{w_{\langle x y\rangle}\right\}$ satisfying

$$
w_{\langle x y\rangle}=w_{\langle y x\rangle}
$$

for all nearest neighbor sites $\langle x y\rangle$ of a $d$-dimensional lattice $\mathbb{Z}^{d}$. The so-called symmetric medium has been considered by several authors (see e.g. [ABSOГ AKSГ AVГMFGWГKuГPV] and references therein).

Except for a basic lemmaTthe general scheme of our presentation will be dimensional independent. However 5 the one-dimensional problem plays a central role in this work since $\Gamma$ in this case $\Gamma$ the macroscopic homogeneity condition can be read from an explicit formula.

The $d=1$ case has been mostly investigated. The first mathematical results [KKSГSo $\Gamma \mathrm{Si}]$ were concerned with asymmetric random walks with transition probabilities $w_{x, x+1}=1-w_{x, x-1} \Gamma x \in \mathbb{Z} \Gamma$ being independent and identically distributed (i.i.d.) random variables (note that $w_{x, x+1} \neq w_{x+1, x}$ ). The trajectories $\left\{X(t), t>0 \mid X(0)=x_{0}\right\}$ of asymmetric random walks were shown to behave very anomalously. Symmetric random walks began to be discussed in a series of papers (see e.g. [АВОГВSWГАВSO]) in connection with the problem of disordered chains of harmonic oscillators (see $[\mathrm{LM}]$ for an introduction and a selection of reprints) and other problems in physics. Using Dyson's integral equation $[\mathrm{D}] \Gamma$ these authors derived the following asymptotic behavior for the trajectories: if

$$
\mathbb{E} w_{x, x+1}^{-1}<\infty
$$

where $\mathbb{E}$ denotes the expectation value with respect to the i.i.d. random variables $\left\{w_{x, x+1}\right\} \Gamma$ then

$$
\mathbb{E} X^{2}(t) \sim 2\left(\mathbb{E} w_{x, x+1}^{-1}\right)^{-1} t,
$$

as $t \rightarrow \infty \Gamma$ and the convergence of $X(t) / \sqrt{t}$ to the Gaussian random variable with zero mean and variance $2\left(\mathbb{E} w_{x, x+1}^{-1}\right)^{-1}$ is implied. In addition if condition (I.2) is violated $\Gamma$ then $\mathbb{E} X^{2}(t)$ grows as $t^{\delta}$ with an exponent $\delta<1$ depending on the divergence of the distribution at $w_{x, x+1}=0$.

A mathematical proof of convergence to Brownian motion for $d=1$ symmetric random walks was 
given by Anshelevich and Vologodski [AV]. For $d \geq 2$ there are at least two different proofs and both require macroscopic homogeneity conditions more stringent than (I.2). Anshelevich KKhanin and Sinai [AKS] proved the result by developing an expansion for the expected value of the inverse of a non-homogeneous discrete Laplacian. Künnemann [Ku] has proven this result by extending Papanicolaou-Varadhan's approach [PV]. Whether (I.2) is a necessary (and sufficient) macroscopic homogeneity condition for $d \geq 2$ Tremains $\Gamma$ to our knowledge an open problem.

The present paper is inspired by the work of Anshelevich KKhanin and Sinai. We use the logic of this proof in order to simplify the Anshelevich and Vologodski's proofs for $d=1$. Our proof $\Gamma$ in particularГeliminates the technical hypothesis of $w_{x, x+1}$ to be strictly positive and illustrates with textbook's mathematical methods the macroscopic homogeneity condition (I.2).

A Brownian motion is described by the diffusion equation. The random environment induces an effective diffusive matrix $\kappa$ whose elements are given by $\lim _{t \rightarrow \infty} \mathbb{E}\left(X_{i}(t) X_{j}(t)\right) / t \Gamma i, j=1, \ldots, d$. For the onedimensional problem $\Gamma$ the reciprocal of this constant is given by the macroscopic homogeneity condition: $\kappa^{-1}=\mathbb{E} w_{x, x+1}^{-1}$. For $d \geq 2 \Gamma$ the two available formulas for the effective diffusion matrix (see [AKSГKu]) do not explicitly show how macroscopic homogenization takes place and this makes it difficult to obtain estimates. In this review (see also [AKS]) the upper bound

$$
\kappa \leq \mathbb{E} w_{\langle x y\rangle}
$$

will be shown to hold if $\left|1-w_{\langle x y\rangle} / \mathbb{E} w_{\langle x y\rangle}\right| \leq \delta<1 / 2$. We also discuss how a lower bound can be obtained using the electrostatic equivalence of the diffusion problem as formulated in [AKS].

The outline of the present work is as follows. In Section II we formulate the problem and state our results. The proofs will be given in Section III under the assumption that the eigenvalues and eigenvectors of the semi-group generator of the process converge to the eigenvalues and eigenfunctions of a Laplacian. The eigenvalue problem is a consequence of our basic lemma (Lemma III.1) which will be proven in Section IV for $d=1$ by Green's function method. The spectral perturbation theory will be presented in Section V. Finally $\mathrm{Tthe}$ diffusion coefficient will be examined in Section VI.

\section{Statement of Results}

Let $\mathbb{B}$ denote the set of bonds of the regular lattice $\mathbb{Z}^{d}$ and let $w=\left\{w_{b}\right\}_{b \in \mathbb{B}}$ be an assignment of positive numbers. Each component $w_{b}$ represents the transition rate of a random walk to go from the site $x$ to $y$ along the bond $b=\langle x y\rangle$. The assignment $w$ defines an symmetric environment on $\mathbb{Z}^{d}$ if the transition rates satisfy $w_{\langle x y\rangle}=w_{\langle y x\rangle}$.

Given an environment $w$ and a finite set $\Lambda \subset \mathbb{Z}^{d} \Gamma$ a continuous time random walk on $\Lambda \Gamma$ with absorbing boundary condition $\Gamma$ is a Markov process $\left\{X_{\Lambda, w}(t), t \geq\right.$ $0\}$ with differential transition matrix $W_{\Lambda}=W_{\Lambda}(w)$ defined by

$$
\begin{aligned}
\left(W_{\Lambda} u\right)_{x} & =\sum_{\substack{y \in \mathbb{Z} d_{:} \\
|x-y|=1}} w_{\langle x y\rangle} u_{y} \\
& =\left(\Delta_{\Lambda, w} u\right)_{x}+u_{x} \sum_{\substack{y \in \mathbb{Z} d_{:} \\
|x-y|=1}} w_{\langle x y\rangle},
\end{aligned}
$$

where

$$
\left(\Delta_{\Lambda, w} u\right)_{x}=\sum_{\substack{y \in \mathbb{Z} d_{:} \\|x-y|=1}} w_{\langle x y\rangle}\left(u_{y}-u_{x}\right),
$$

for all $x \in \Lambda$ and $u$ such that $u_{y}=0$ if $y \in \mathbb{Z}^{d} \backslash \Lambda$.

Note that $-\Delta_{\Lambda, w}$ is a positive matrix $\Gamma$

$$
\left(u,\left(-\Delta_{\Lambda, w}\right) u\right)=\frac{1}{2} \sum_{\substack{x, y \in \mathbb{Z} d_{:} \\|x-y|=1}} w_{\langle x y\rangle}\left(u_{y}-u_{x}\right)^{2} \geq 0,
$$

and $\Gamma$ if $w_{b}=\bar{w}$ for all $b \in \mathbb{B} \Gamma \Delta_{\Lambda, \bar{w}}=\bar{w} \Delta_{\Lambda}$ where $\Delta_{\Lambda}$ is the finite difference Laplacian with 0 -Dirichlet boundary condition on $\Lambda$. From here on $\Gamma \Lambda$ is taken to be the hypercube centered at origin with size $|\Lambda|=(2 L-1)^{d} \Gamma$ $L \in \mathbb{N}: \Lambda_{L}:=\left\{x=\left(x_{1}, \ldots, x_{d}\right) \in \mathbb{Z}^{d}: \sup _{i}\left|x_{i}\right|<L\right\} \Gamma$ and all quantities depending on $\Lambda$ will be indexed by $L$ instead.

The probability distribution of $\left\{X_{L, w}(t), t \geq 0\right\}$ is governed by the semi-group $\widehat{T}_{t}^{L}$ generated by $\Delta_{L, w}$. If $X_{L, w}(t)$ denotes the position of a random walk at time $t \Gamma$ then

$$
P\left(X_{L, w}(t)=x \mid X_{L, w}(0)=0\right)=\left(e^{t \Delta_{L, w}} u_{0}\right)_{x},
$$

where $\left(u_{0}\right)_{x}=\delta_{0, x}$.

The semi-group $\widehat{T}_{t}^{L}$ is the solution of the initial value problem in $\mathbb{R}^{\Lambda} \Gamma$

$$
\frac{d u}{d t}-\Delta_{L, w} u=0,
$$

with initial condition $u(0)=u_{0}$. 
The solution of (II.4) exists for all times $t>0$ and all sizes $L<\infty$ but may depend on the realization of $w$ and on the initial value. We present the sufficient conditions on the environment $w$ by which the solution of (II.4) Tunder suitable scaling of time and space $\Gamma$ converges almost everywhere in $w$ to the fundamental solution of the heat equation $\Gamma$

$$
\frac{\partial u}{\partial t}-\partial^{2} u=0
$$

with $u(t, \partial \mathcal{D})=0$. Here $\Gamma$ (II.5) is defined in the domain $t>0$ and $\xi \in \mathcal{D}:=(-1,1)^{d}$ with boundary $\partial \mathcal{D}=\left\{\xi: \sup _{i}\left|\xi_{i}\right|=1\right\} \Gamma$ and $\partial^{2}=\partial^{2}(\kappa)$ is given by

$$
\partial^{2}=\partial \cdot \kappa \partial=\sum_{i, j=1}^{d} \kappa_{i j} \frac{\partial^{2}}{\partial_{\xi_{i}} \partial_{\xi_{j}}} .
$$

The heat kernel $T_{t}(\eta, \zeta)=e^{t \partial^{2}}(\eta, \zeta)$ when defined in $\mathbb{R}^{d} \times \mathbb{R}^{d}$ gives rise to a Wiener process (or Brownian motion) $\{B(t), t \geq 0\}$ with covariance $\mathbb{E} B(t) B(s)=$ $4 \kappa \min (s, t)$ (see e.g. Simon [S]). In view of the boundary condition $\Gamma T_{t}$ yields a Brownian motion $\left\{B_{0}(t), t \geq\right.$ $0\}$ on the domain $\mathcal{D}$ with absorbing frontiers. The probability density of $B_{0}(s+t)-B_{0}(s)$ to be equal to $\xi$ can be obtained by solving equations (II.5) by Fourier method

$$
T_{t}(\xi, 0)=\sum_{n \in \mathbb{N}_{+}^{d}} e^{-\left(\pi^{2} / 4\right) n^{2} t} \prod_{i=1}^{d} \operatorname{sc}\left(\pi n_{i} \xi_{i} / 2\right)
$$

where $n^{2}=n \cdot \kappa n=\sum_{i, j=1}^{d} n_{i} \kappa_{i j} n_{j}$ and $\operatorname{sc}\left(n_{i} x\right)$ stands for either $\cos \left(n_{i} x\right)$ or $\sin \left(n_{i} x\right)$ depending on whether $n_{i}$ is an odd or even number.

This discussion suggests the following definition.

Definition II.1 The random walk in a random environment $w$ is said to converge to a Brownian motion if there exist a constant $\kappa=\kappa(w)$, the diffusion coefficient, such that

$$
\lim _{L \rightarrow \infty}\left(e^{L^{2} t \Delta_{L, w}} u_{0}\right)_{[L \xi]}=\left(e^{t \partial^{2}(\kappa)} \mu_{0}\right)(\xi)
$$

uniformly in $t>0, \xi \in \mathcal{D}$ and $\mu_{0}$ on the space of finite measures $\mathcal{M}$ with support in $\mathcal{D}$. For $r \in \mathbb{R}^{d},[r] \in \mathbb{Z}^{d}$ and has as components the integer part of the components of $r$; and $u_{0} \in \mathbb{R}^{\Lambda_{L}}$ is a vector given by

$$
\left(u_{0}\right)_{x}=L^{d} \int_{x_{1} / L}^{\left(x_{1}+1\right) / L} \cdots \int_{x_{d} / L}^{\left(x_{d}+1\right) / L} d \mu_{0}(\zeta) .
$$

It is important to note that $\Gamma$ by the dominant convergence theorem $\Gamma$ this definition implies the convergence in distribution of the random walk process $\left\{(1 / L) X_{L, w}\left(L^{2} t\right), t \geq 0\right\}$ to the Brownian motion $\left\{B_{0}(t), t \geq 0\right\}$ as it is known in Probability Theory (weak convergence of their distributions): $X_{L}(t) \equiv$ $\frac{1}{L} X_{L, w}\left(L^{2} t\right) \longrightarrow B_{0}(t)$ in distribution if $\Gamma$ for any collection $0<s_{1}<\cdots<s_{n}$ of positive numbers and any collection $f_{1}, \ldots, f_{n}$ of bounded and continuous functions in $\mathcal{D} \Gamma n \in \mathbb{N} \Gamma$ we have

$$
\mathbb{E}_{\mu_{0}} f_{1}\left(X_{L}\left(s_{1}\right)\right) \cdots f_{n}\left(X_{L}\left(s_{n}\right)\right) \longrightarrow \mathbb{E}_{\mu_{0}} f_{1}\left(B_{0}\left(s_{1}\right)\right) \cdots f_{n}\left(B_{0}\left(s_{n}\right)\right),
$$

as $N \rightarrow \infty \Gamma$ where $\mathbb{E}_{\mu_{0}}$ means the expectation of the process starting with the measure $\mu_{0}$. Note also that $X_{L}(t)$ has been scaled as in the central limit theorem: $X_{L}(t)$ is a sum of about $L^{2}$ independent increments ${ }^{1}$ divided up by $L$.

\section{Theorem II.2 (Anshelevich and Vologodski) If} $d=1$ and the environment $w$ is a stationary process such that the partial sums

$$
s_{x}(w)=\frac{1}{x} \sum_{z=0}^{x-1} \frac{1}{w_{z, z+1}}
$$

converge as $x \rightarrow \infty$ to $\kappa^{-1}, 0<\kappa<\infty$, almost everywhere in $w$, then a random walk in a random environment $w$ converges, for almost every $w$, to a Brownian motion with diffusion coefficient $\kappa$.

\footnotetext{
${ }^{1}$ A simple random walk with continuum time jumps according to a Poisson process on time with rate $2 d$ and there will be $2 d L^{2} t$ jumps in average after a time $L^{2} t$. With the random environment, the Poisson process has a site dependent rate given by $\sum_{y:|x-y|=1} w_{\langle x y\rangle}$.
} 
Theorem II.3 (Anshelevich, Khanin and Sinai)

For any $d \geq 1$ and $\delta<1 / 2$, let $w=\left\{w_{b}\right\}_{b \in \mathbb{B}}$ be independent and identically distributed random variables such that

$$
\left|1-\frac{w_{b}}{\bar{w}}\right| \leq \delta
$$

with $\bar{w}=\mathbb{E} w_{b}$. Then a random walk in a random environment $w$ converges, for almost every $w$, to a Brownian motion with diffusion coefficient matrix $\kappa=$ $\kappa(d, \delta, \bar{w})$.

Remark II.4 1. Theorem II.2 was proven in [AV] under the condition (II.9) with $w_{x, x+1}>0$. The positive condition has been eliminated in our proof. Condition (II.9) is met if $w=\left\{w_{b}\right\}$ are $i . i$ d. random variables with $0<\mathbb{E} w_{b}^{-1}<\infty$. Finiteness of first negative moment seems to be, according to arguments presented in [ABSO] (see also [FIN]), a sufficient and necessary homogeneity condition since, otherwise, the walk would spend a extremely large time between jumps leading the process to be sub-diffusive.

2. Whether the homogeneity condition $0<\mathbb{E} w_{b}^{-1}<$ $\infty$ is also sufficient for $d>1$ is, to our knowledge, an open problem. It would already be an important achievement to prove Theorem II.3 for any distribution whose support is $\mathbb{R}$. It is unfortunate that both proofs (see $[A K S, K u]$ ) require as a technical step $w_{b}$ to be bounded away from 0 and $\infty$.

3. Theorem II.3 holds also if the transition probabilities $\left\{w_{x y}\right\}$ do no vanish for $y-x$ belonging to a finite set $\mathfrak{U}^{+}$that is able to generate $\mathbb{Z}^{d}$ by translations. Under the same sort of condition (II.10), [AKS] have shown convergence to Brownian motion satisfying the diffusion equation (II.5).

4. Theorem II.3 can also be extended to Brownian motion on $\mathbb{R}^{d}$ if one combines the result with both absorbing and periodic boundary conditions on $\partial \mathcal{D}$ (see details in [AKS]).
Theorem II.5 Under the conditions of Theorem II.3 and a conjecture formulated in (VI.27), there exist a finite constant $C=C(d)$, such that the bounds

$$
1-\varrho \leq \frac{\kappa}{\mathbb{E} w_{b}} \leq 1
$$

hold with 1 being the $d \times d$ identity matrix and $\varrho$ a positive matrix such that

$$
\varrho \leq \frac{4 \delta C(1+C)}{1-4 \delta(1+C)}
$$

in the sense of quadratic forms.

\section{Basic Lemma}

The proof of Theorems II.2 and II.3 presented in this section are based on the uniform convergence of the eigenvalues and eigenvectors of $\Delta_{L, w}$ to the eigenvalues and eigenfunctions of the Laplacian operator $\partial^{2}$ on $\mathcal{D}$. The uniform convergence follows from a classical result in perturbation theory which says the following.

If $A_{1}, A_{2}, \ldots, A_{n}, \ldots$ is a sequence of bounded operators in a Hilbert space $\mathcal{H}$ which converge to $A$ in the operator norm $\Gamma$ then all isolated pieces of their spectrum and their respective projections converge uniformly $\Gamma$ as $n \rightarrow \infty$ Tto those of $A$.

Because $\Delta_{L, w}$ and $\partial^{2}$ are unbounded operators we consider their inverse instead. To formulate the results of this section $\Gamma$ we need some definitions.

Let $i_{L}$ be an isometry of the vector space $\mathbb{R}^{\Lambda}$ into the piecewise constant functions in the vector space $L_{0}^{2}(\mathcal{D}) \Gamma$ of square-integrable functions $f$ on $\mathcal{D}=(-1,1)^{d}$ with $f(\partial \mathcal{D})=0$ :

$$
i_{L}: \mathbb{R}^{\Lambda} \longrightarrow L_{0}^{2}(\mathcal{D})
$$

given by

$$
\left(i_{L} u\right)(\xi)= \begin{cases}u_{[L \xi]} & \text { if } \quad \xi \in(-1,1)^{d} \\ 0 & \text { if } \quad \xi \in \partial \mathcal{D}\end{cases}
$$

with $[x]$ as in Definition II.1.

The adjoint operator $i_{L}^{\dagger}: L_{0}^{2}(\mathcal{D}) \longrightarrow \mathbb{R}^{\Lambda} \Gamma$

$$
\left(i_{L}^{\dagger} f\right)_{x}=L^{d} \int_{x_{1} / L}^{\left(x_{1}+1\right) / L} d x_{1} \cdots \int_{x_{d} / L}^{\left(x_{d}+1\right) / L} d x_{d} f(x)
$$


is defined by the equation

$$
\left\langle f, i_{L} u\right\rangle=\left(i_{L}^{\dagger} f, u\right)
$$

with the inner product in $\mathbb{R}^{\Lambda}$ and $L_{0}^{2}(\mathcal{D})$ given $\Gamma$ respectively $\mathrm{rby}$

$$
(u, v)=\frac{1}{L^{d}} \sum_{x \in \Lambda} u_{x} v_{x}
$$

and

$$
\langle f, g\rangle=\int_{[-1,1]^{d}} f(x) g(x) d x .
$$

We shall denote by

$$
\Xi_{L}^{-1}:=L^{-2}\left(i_{L} \Delta_{L, w}^{-1} i_{L}^{\dagger}\right)
$$

the scaled inverse of $\Delta_{L, w}$. The operator kernel of $\Xi_{L}^{-1}$ is a step function with step-width $1 / L$ which $\Gamma$ as we shall see in the next lemma $\Gamma$ approximates the kernel $\left(\partial^{2}\right)^{-1}(r, s)$ in the operator norm induced by $L_{0}^{2}-$ norm:

$$
\|A\|:=\sup _{f:\|f\|_{2}=1}\|A f\|_{2},
$$

where $\|f\|_{2}^{2}:=\langle f, f\rangle$.

Lemma III.1 (Basic Lemma) Under the conditions of Theorems II.3 and II.3, there exist a finite number $L_{0}=L_{0}(w)$ such that, $\left\{\Xi_{L}^{-1} ; L>L_{0}\right\}$ is a sequence of bounded self-adjoint operator in $L_{0}^{2}(\mathcal{D})$ which converges

$$
\left\|\Xi_{L}^{-1}-\left(\partial^{2}\right)^{-1}\right\| \longrightarrow 0
$$

as $L \rightarrow \infty$, in the operator norm topology. $[\mathrm{F}])$ :

It thus follows from perturbation theory (see e.g.

Corollary III.2 If $\lambda$ is an isolated eigenvalue of $\left(\partial^{2}\right)^{-1}$ and $E$ the orthogonal projection in its eigenspace $\mathcal{E}$, one can find a subspace $\mathcal{E}^{L} \subset L_{0}^{2}(\mathcal{D})$, invariant under $\Xi_{L}^{-1}$, and a corresponding orthogonal projection $E^{L}$ such that

$$
\left\|E^{L}-E\right\| \longrightarrow 0
$$

and

$$
\left\|E^{L}\left(\Xi_{L}^{-1}-\lambda I\right) E^{L}\right\| \longrightarrow 0
$$

as $L \rightarrow \infty$
Lemma III.1 will be proven for $d=1$ random walks in Section IV. This lemma reduces the Brownian motion limit problem to the convergence of the inverse matrix $\Delta_{L, w}^{-1}$ to the inverse Laplacian $\left(\partial^{2}\right)^{-1}$ in the $L_{0}^{2}$ operator norm topology. Corollary III.2 will be proven in Section V.

Proof of Theorems II.2 and II.3 assuming Corollary III.2. (As in Appendix 3 of $[\mathrm{AKS}]$ ) Let $\varphi: \mathbb{R}_{-} \longrightarrow \mathbb{R}$ be given by

$$
\varphi(\lambda)=e^{t / \lambda}
$$

and note that $\varphi$ is uniformly continuous at $\lambda=0$ with $\varphi(0)=0$. We have $T_{t}=\varphi\left(\left(\partial^{2}\right)^{-1}\right)$ and $T_{t}^{L}=e^{t \Xi_{L}}=$ $\varphi\left(\Xi_{L}^{-1}\right)$.

In view of Definition II. 1 and the isometry $i_{L} \Gamma$ Theorems II.2 and II.3 can be restated as ${ }^{2}$

$$
\sup _{\mu_{0} \in \mathcal{M}} \sup _{\xi \in \mathcal{D}}\left|\left[\left(T_{t}-T_{t}^{L}\right) \mu_{0}\right](\xi)\right| \longrightarrow 0
$$

as $L \rightarrow \infty$. We shall prove an equivalent statement:

$$
\lim _{L \rightarrow \infty}\left\|T_{t}-T_{t}^{L}\right\|=\lim _{L \rightarrow \infty}\left\|\varphi\left(\left(\partial^{2}\right)^{-1}\right)-\varphi\left(\Xi_{L}^{-1}\right)\right\|=0
$$

The inverse Laplacian $\left(\partial^{2}\right)^{-1}$ on $\mathcal{D}$ with 0 -Dirichlet boundary condition is a compact operator with spectral decomposition given by (recall equation (II.7))

$$
\left(\partial^{2}\right)^{-1}=\sum_{n \in \mathbb{N}_{+}^{d}} \lambda_{n} E_{n}
$$

where $\lambda_{n}=\left((\pi / 2)^{2} \sum_{i, j=1}^{d} n_{i} \kappa_{i, j} n_{j}\right)^{-1} \equiv 4 /\left(\pi^{2} n^{2}\right)$ and $e_{n}(\xi)=\prod_{i=1}^{d} \operatorname{sc}\left[(\pi / 2) n_{i} \xi_{i}\right] \Gamma n \in \mathbb{N}_{+}^{d}$ Tare eigenvalues and associate eigenfunctions of $\left(\partial^{2}\right)^{-1}$ and

$$
E_{n} f=\left\langle e_{n}, f\right\rangle e_{n}
$$

Because 0 is an accumulation point $\Gamma$ we introduce an integer cut-off $N<\infty$ and let

$$
\left(\partial_{N}^{2}\right)^{-1}=\sum_{\substack{n \in \mathbb{N}^{d} \\|n| \leq N}} \lambda_{n} E_{n}
$$

\footnotetext{
${ }^{2}$ The isometry $i_{L}$ has been introduced to bring all operators to the same Hilbert space $L_{0}^{2}(\mathcal{D})$. Note, however, that $\Xi_{L}^{-1}$ and $T_{t}^{L}$ remain finite rank operators.
} 
We have

$$
\left\|\varphi\left(\left(\partial_{N}^{2}\right)^{-1}\right)-\varphi\left(\left(\partial^{2}\right)^{-1}\right)\right\|^{2}=\sum_{|n|>N} \varphi^{2}\left(\lambda_{n}\right),
$$

which can be made as small as we wish by letting $N \rightarrow \infty$. More generally $\Gamma$ the uniform continuity of $\varphi$ at 0 means the following: given $\varepsilon>0$ and a nonpositive bounded operators $A \Gamma$ we can find $\delta>0$ such that if $\|A\|<\delta$ we have $\|\varphi(A)\|<\varepsilon / 3$. We shall use this fact often in the sequel.
From Corollary III.2Гthere exist a projector

$$
E^{L, N}=\sum_{\substack{n \in \mathbb{N}^{3}: \\|n| \leq N}} E_{n}^{L},
$$

where $E_{n}^{L}$ is $\Gamma$ analogously to $E_{n} \Gamma$ the projector on the invariant subspace: $\Xi_{L}^{-1} \mathcal{E}_{n}^{L}=\mathcal{E}_{n}^{L}$. Writing

$$
\Xi_{L, N}^{-1}=E^{L, N} \Xi_{L}^{-1} E^{L, N}
$$

and using the fact that $E^{L, N}$ is an orthogonal projector $\Gamma$ we have

$$
\begin{aligned}
\varphi\left(\Xi_{L}^{-1}\right)-\varphi\left(\Xi_{L, N}^{-1}\right) & =\varphi\left(\Xi_{L, N}^{-1}+\left(\Xi_{L}^{-1}-\Xi_{L, N}^{-1}\right)\right)-\varphi\left(\Xi_{L, N}^{-1}\right) \\
& =\varphi\left(\Xi_{L}^{-1}-\Xi_{L, N}^{-1}\right)
\end{aligned}
$$

(here $\varphi\left(A^{-1}\right)$ is defined by its power series $I+t A+$ $\left.t^{2} A^{2} / 2+\cdots\right)$.

We now show that $\left\|\Xi_{L}^{-1}-\Xi_{L, N}^{-1}\right\|$ can be made smaller then $\delta=\delta(\varepsilon)$ if $L$ and $N$ are chosen sufficient large. By Lemma III.1 $\Gamma$ there exist $L_{1} \geq L_{0} \Gamma$ $L_{1}=L_{1}(\delta) \Gamma$ such that

$$
\left\|\Xi_{L}^{-1}-\left(\partial^{2}\right)^{-1}\right\|<\frac{\delta}{3}
$$

for all $L>L_{1}$. From (III.12) and (III.14) $\Gamma$ there exist
$N_{1}=N_{1}(\delta)$ such that

$$
\left\|\left(\partial^{2}\right)^{-1}-\left(\partial_{N}^{2}\right)^{-1}\right\|<\frac{\delta}{3},
$$

if $N>N_{1}$. By Lemma III.1 $\Gamma$ there exist $L_{2}>L_{0} \Gamma$ $L_{2}=L_{2}(\delta) \Gamma$ such that

$$
\left\|\left(\partial_{N}^{2}\right)^{-1}-\Xi_{L, N}^{-1}\right\|<\frac{\delta}{3}
$$

holds for all $L>L_{2}$ with $N$ fixed.

If $L>\max \left(L_{1}, L_{2}\right)$ and $N>N_{1}$ Tequations (III.18)(III.20) yield

$$
\left\|\Xi_{L}^{-1}-\Xi_{L, N}^{-1}\right\| \leq\left\|\Xi_{L}^{-1}-\left(\partial^{2}\right)^{-1}\right\|+\left\|\left(\partial^{2}\right)^{-1}-\left(\partial_{N}^{2}\right)^{-1}\right\|+\left\|\left(\partial_{N}^{2}\right)^{-1}-\Xi_{L, N}^{-1}\right\|<\delta,
$$

which implies $\Gamma$ due the continuity of $\varphi$ and the orthogonal relation (III.17) $\Gamma$

$$
\left\|\varphi\left(\Xi_{L}\right)-\varphi\left(\Xi_{L, N}\right)\right\|<\frac{\varepsilon}{3} .
$$

By uniform continuity of $\varphi$ and (III.20) $\Gamma$ we also have

$$
\left\|\varphi\left(\left(\partial^{2}\right)^{-1}\right)-\varphi\left(\left(\partial_{N}^{2}\right)^{-1}\right)\right\|<\frac{\varepsilon}{3} .
$$

In addition $\Gamma$ using the spectral decomposition of
$\left(\partial_{N}^{2}\right)^{-1}$ and $\Xi_{L, N}^{-1}$ Tand taking into account

$$
\left\|\lambda_{n} E_{n}-\lambda_{n}^{L} E_{n}^{L}\right\| \leq\left|\lambda_{n}-\lambda_{n}^{L}\right|\left\|E_{n}\right\|+\left|\lambda_{n}^{L}\right|\left\|E_{n}-E_{n}^{L}\right\|
$$

and Lemma III.1 $\Gamma$ we can find $L_{3}>L_{0} \Gamma L_{3}=L_{3}(\varepsilon, N) \Gamma$ such that

$$
\left\|\varphi\left(\left(\partial_{N}^{2}\right)^{-1}\right)-\varphi\left(\Xi_{N, L}^{-1}\right)\right\|<\frac{\varepsilon}{3} .
$$

Now $\Gamma$ let $L>\max \left(L_{1}, L_{2}, L_{3}\right)$. It then follows from (III.22) Г(III.23) and (III.25) 


$$
\begin{aligned}
\left\|\varphi\left(\left(\partial^{2}\right)^{-1}\right)-\varphi\left(\Xi_{L}^{-1}\right)\right\| \leq & \left\|\varphi\left(\left(\partial^{2}\right)^{-1}\right)-\varphi\left(\left(\partial_{N}^{2}\right)^{-1}\right)\right\| \\
& +\left\|\varphi\left(\left(\partial_{N}^{2}\right)^{-1}\right)-\varphi\left(\Xi_{N, L}^{-1}\right)\right\|+\left\|\varphi\left(\Xi_{L}^{-1}\right)-\varphi\left(\Xi_{L, N}^{-1}\right)\right\|<\varepsilon,
\end{aligned}
$$

which implies strong convergence of the semi-group and completes the proof of Theorem II.3.

Remark III.3 The introduction of the cut-off $N$ in (III.14) is necessary even for homogeneous environment. In this case, the eigenvalues $\lambda_{n}^{L}$ and eigenvectors $e_{n}^{L}$ of $L^{-2} \Delta_{L}^{-1}$ can be computed explicitly:

$$
\lambda_{n}^{L}=\left(-4 L^{2} \sum_{i=1}^{d} \sin ^{2} \frac{\pi}{4 L} n_{i}\right)^{-1}
$$

and

$$
e_{n}^{L}(x)=\prod_{i=1}^{d} \mathrm{sc}\left(\frac{\pi}{2 L} n_{i} x_{i}\right),
$$

with $n \in \Lambda^{*}:=\{1,2, \ldots,(2 L-1)\}^{d}$ (recall $\operatorname{sc}\left(n_{i} x\right)$ stands for $\cos \left(n_{i} x\right)$ or $\sin \left(n_{i} x\right)$, depending on whether $n_{i}$ is an odd or even number). Note that $\left|\lambda_{n}-\lambda_{n}^{L}\right|$, with $\lambda_{n}$ given by (III.12), may not be small if $|n|=O(L)$. We always pick $N$ large but fixed and let $L \rightarrow \infty$ in order (III.25) to be true.

\section{Proof of Lemma III.1 $(d=1)$}

In this section we prove Lemma III. 1 for $d=1$. We consider the second-order Sturm-Liouville difference equation

$$
\Delta_{L, w} u=f
$$

with $u(\partial \mathcal{D})=0$ Гand use the method of Green to calculate the matrix elements of $\Delta_{L, w}^{-1}$. This gives $\Gamma i n$ view of equation (III.5) $\Gamma$ an explicit formula for the operator kernel $\Xi_{L}^{-1}(r, s)$.

The procedure starts by looking for two linear independent solutions of the homogeneous equation

$$
\left(\Delta_{L, w} u\right)_{x}=w_{\langle x-1, x\rangle}\left(u_{x-1}-u_{x}\right)-w_{\langle x, x+1\rangle}\left(u_{x}-u_{x+1}\right)=0,
$$

with $x \in\{-L+1, \ldots, L-1\}$ and $u_{-L}=u_{L}=0$. Without loss of generality $\Gamma$ we set $w_{\langle L-1, L\rangle}=w_{\langle-L,-L+1\rangle}=$ $\kappa$.

Proposition IV.1 Let $\xi_{L} \in \mathbb{R}^{2 L-1}$ be a vector valued function of the environment $w$ given by

$$
\left(\xi_{L}\right)_{x}=\eta_{L} \sum_{y=-L+1}^{x} w_{\langle y-1, y\rangle}^{-1},
$$

for all $x \in\{-L+1, \ldots, L-1\}$, where

$$
\eta_{L}^{-1}=\sum_{y=-L+1}^{L} w_{\langle y-1, y\rangle}^{-1} .
$$

Then $u_{1}=\xi_{L}$ and $u_{2}=1-\xi_{L}$ are two linear independent solutions of (IV.1).
Proof. $u_{1}=\xi_{L}$ is a solution of (IV.1) by simple verification and the same can be said of $u_{2}=1-\xi_{L}$. For this nnote that

$$
w_{\langle x-1, x\rangle}\left(\nabla \xi_{L}\right)_{x}=\eta_{L}
$$

holds uniformly in $x \Gamma$ where $(\nabla u)_{x}=u_{x}-u_{x-1}$. It thus remains to verify that they are linear independent.

Let $W=W\left(u_{1}, u_{2} ; x\right)$ be the "Wronskian" of the two solutions $u_{1}$ and $u_{2}$ given by the following determinant

$$
W=\left|\begin{array}{cc}
\left(u_{1}\right)_{x} & \left(u_{2}\right)_{x} \\
w_{\langle x-1, x\rangle}\left(\nabla u_{1}\right)_{x} & w_{\langle x-1, x\rangle}\left(\nabla u_{2}\right)_{x}
\end{array}\right| .
$$

It follows that two solutions $u_{1}$ and $u_{2}$ are linear independent if $W\left(u_{1}, u_{2} ; x\right) \neq 0$ for all $x \in\{-L, \ldots, L\}$. 
Plugging $u_{1}$ and $u_{2}$ into (IV.4) we have $\Gamma$ in view of (IV.3) Г

$$
W=-\eta_{L}\left[\left(\xi_{L}\right)_{x}+1-\left(\xi_{L}\right)_{x}\right]=-\eta_{L},
$$

which concludes the proof of the proposition. so called Green's function method (see e.g. $[\mathrm{J}]$ ):

$$
\left(\Delta_{L, w}^{-1}\right)_{x, y}=\left\{\begin{array}{lll}
\frac{\left(\xi_{L}\right)_{x}\left[1-\left(\xi_{L}\right)_{y}\right]}{-\eta_{L}} & \text { if } & x \leq y \\
\frac{\left[1-\left(\xi_{L}\right)_{x}\right]\left(\xi_{L}\right)_{y}}{-\eta_{L}} & \text { if } & x>y .
\end{array}\right.
$$

To see this is true $\Gamma$ we note $\left(\Delta_{L, w}^{-1}\right)_{z, y}$ is the $z-$ component of a vector for each $y$ fixed. So tion

$$
\left(\Delta_{L, w} \Delta_{L, w}^{-1}\right)_{x, y}=0
$$

holds for all $x \neq y$. For $x=y$ we have

$$
\begin{aligned}
\left(\Delta_{L, w} \Delta_{L, w}^{-1}\right)_{x, x} & =w_{\langle x, x+1\rangle}\left(\nabla \Delta_{L, w}^{-1}\right)_{x+1, x}-w_{\langle x-1, x\rangle}\left(\nabla \Delta_{L, w}^{-1}\right)_{x, x} \\
& =-w_{\langle x, x+1\rangle}\left(\nabla \xi_{L}\right)_{x+1} \frac{\left(\xi_{L}\right)_{x}}{-\eta_{L}}-w_{\langle x-1, x\rangle}\left(\nabla \xi_{L}\right)_{x} \frac{1-\left(\xi_{L}\right)_{x}}{-\eta_{L}} \\
& =\left(\xi_{L}\right)_{x}+\left(1-\left(\xi_{L}\right)_{x}\right)=1
\end{aligned}
$$

by (IV.3) Гverifying the assertion.

We are now ready to write the operator kernel of $\Xi_{L}^{-1}$. In view of

$$
\begin{aligned}
\left(i^{\dagger} g, A i^{\dagger} f\right) & =\frac{1}{L} \sum_{x, y \in \Lambda}\left(i^{\dagger} g\right)_{x} A_{x, y}\left(i^{\dagger} f\right)_{y} \\
& =\frac{1}{L} \sum_{x, y \in \Lambda} L \int_{x / L}^{(x+1) / L} d r g(r) A_{x, y} L \int_{y / L}^{(y+1) / L} d s g(s) \\
& =\int_{-1}^{1} d r \int_{-1}^{1} d s g(r)\left(L A_{[L r],[L s]}\right) f(s)=\left\langle g, i A i^{\dagger} f\right\rangle,
\end{aligned}
$$

valid for any $(2 L-1) \times(2 L-1)$ matrix $A$ and $f \in L_{0}^{2}(\mathcal{D}) \Gamma$ and definitions (III.5) and (III.1) $\Gamma$ we have

$$
\Xi_{L}^{-1}(r, s)=L^{-2}\left(i \Delta_{L, w}^{-1} i^{\dagger}\right)(r, s)=L^{-1}\left(\Delta_{L, w}^{-1}\right)_{[L r],[L s]},
$$




$$
\Xi_{L}^{-1}(r, s)=\frac{-1}{4 L \eta_{L}}\left\{1-\left|\left(\zeta_{L}\right)_{[L r]}-\left(\zeta_{L}\right)_{[L s]}\right|-\left(\zeta_{L}\right)_{[L r]}\left(\zeta_{L}\right)_{[L s]}\right\}
$$

in view of the fact that $\left(\zeta_{L}\right)_{x}$ is a monotone increasing function of $x$.

By Schwarz inequality the operator norm (III.6) is bounded by the $L^{2}$-norm of the operator kernel $\Gamma$ the Hilbert-Schmidt norm $|\|K\||^{2}:=$ $\int_{-1}^{1} d r \int_{-1}^{1} d s|K(r, s)|^{2}$. Since the functions in the Hilbert space has compact support $\Gamma$ we have

$$
\|K\| \leq|\|K\|| \leq 4 \sup _{-1<r, s<1}|K(r, s)|
$$

and $\left\|\Xi_{L}^{-1}-\left(\partial^{2}\right)^{-1}\right\| \longrightarrow 0$ is implied by the pointwise convergence $\Xi_{L}^{-1}(r, s) \longrightarrow\left(\partial^{2}\right)^{-1}(r, s)$ of the operator kernel. We shall see that the latter convergence sense is consequence of the following result.

Proposition IV.2 Given $\varepsilon>0$ and $w$ satisfying the hypothesis of Theorem II.2. Then, there exist an integer number $L_{0}=L_{0}(\varepsilon, w)$ such that

$$
\left|\left(\zeta_{L}\right)_{[L r]}-r\right|<\varepsilon
$$

holds for all $L>L_{0}$ and $-1<r<1$.

Proof. Under the hypothesis of Theorem II.2 the strong law of large numbers holds and

$$
2 L \eta_{L} \longrightarrow \kappa,
$$

as $L \rightarrow \infty \Gamma$ for almost every $w$ (see eq. (IV.2)). Analogously $\Gamma$ since $[L r] / L \longrightarrow r$ as $L \rightarrow \infty \Gamma$

$\left(\zeta_{L}\right)_{[L r]}=2 L \eta_{L} \cdot \frac{[L r]+L}{L} \cdot \frac{1}{[L r]+L} \sum_{y=-L+1}^{[L r]} w_{\langle y-1, y\rangle}^{-1}-1$ converges to $r$ as $L \rightarrow \infty$ for each $r \in(-1,1)$ and this gives (IV.10).

The Green's function method can also be used to compute the integral kernel of $\left(\partial^{2}\right)^{-1}$ as an operator in the Hilbert space $L_{0}^{2}(\mathcal{D})$. The two linear independent solutions of the homogeneous equation

$$
\kappa \frac{d^{2} f}{d r^{2}}=0, \quad-1<r<1
$$

with boundary condition $f(-1)=f(1)=0$ are $f_{1}=1+$ $r$ and $f_{2}=1-r$. Replacing $u_{1(2)}$ and $w_{\langle x-1, x\rangle}\left(\nabla u_{1(2)}\right)$ by $f_{1(2)}$ and $\kappa\left(d f_{1(2)} / d r\right)$ in (IV.4) $\Gamma$ gives $W=-2 \kappa$. Substituting these into (IV.6) following the simplification of (IV.9) Гyields

$$
\left(\partial^{2}\right)^{-1}(r, s)=\frac{-1}{2 \kappa}\{1-|r-s|-r s\}
$$

Note that $\left|\left(\partial^{2}\right)^{-1}(r, s)\right| \leq 1 /(2 \kappa)$ and $\Gamma$ in view of (IV.9) and Proposition IV.2Г

$$
\left|\Xi_{L}^{-1}(r, s)\right| \leq C
$$

holds uniformly in $r, s \in(-1,1)$ for all $L>L_{0}$.

Now $\Gamma$ let $\rho_{L}(r):=\left(i \zeta_{L}\right)(r)-r$ and $\widehat{\Xi}_{L}^{-1}:=$ $2 L \eta_{L} \Xi_{L}^{-1} / \kappa$. Then $\Gamma$ if $L>L_{0} \Gamma$ in view of (IV.9) $\Gamma$ (IV.14) and Proposition IV.2Гwe have

$$
\left|\Xi_{L}^{-1}(r, s)-\left(\partial^{2}\right)^{-1}(r, s)\right| \leq\left|\frac{2 L \eta_{L}}{\kappa}-1\right|\left|\Xi_{L}^{-1}(r, s)\right|+\left|\widehat{\Xi}_{L}^{-1}(r, s)-\left(\partial^{2}\right)^{-1}(r, s)\right|,
$$

where 


$$
\begin{aligned}
\left|\widehat{\Xi}_{L}^{-1}(r, s)-\left(\partial^{2}\right)^{-1}(r, s)\right| & =\frac{1}{2 \kappa}\left|\rho_{L}(r) \rho_{L}(s)+r \rho_{L}(s)+s \rho_{L}(r)+\right| \rho_{L}(s)-\rho_{L}(r)|| \\
& <\frac{2 \varepsilon}{\kappa}+\frac{\varepsilon^{2}}{2 \kappa}
\end{aligned}
$$

uniformly in $r, s \in(-1,1)$. When combined with (IV.11) and (IV.15) this proves Lemma III.1 for $d=1$.

\section{Perturbation of Spectra}

Proof of Corollary III.2. This proof can be found in Appendix B of [AKS] and is essentially based on the perturbation theory of Hermitian bounded operators developed by Friedrichs in [F]. Since it can be described shortly $\Gamma$ we repeat the proof's derivation for completeness. Our derivation $\Gamma$ however $\Gamma$ is more close to $[\mathrm{F}]$ in the sense that we perturb an interval of the spectrum. When the interval contains one single eigenvalue this reduces to the derivation of [AKS]. The generalization is however essential in dealing with intervals containing accumulation point of the spectrum. This situation has to be considered in order to show that the spectrum projection in such intervals remains orthogonal when the perturbation is turned on.

We now introduce some notation. Let $I_{0} \in \mathbb{R}$ be an isolated closed interval of the spectrum $\sigma\left(\partial^{-2}\right)$ of $\partial^{-2}$ defined with Dirichlet boundary condition on $\mathcal{D}=(-1,1)$. There exist $0<\delta<\infty$ and an interval $I \subset I_{0}$ such that $I \cap \sigma\left(\partial^{-2}\right)=\sigma\left(\partial^{-2}\right) \cap I_{0}$ and

$$
\operatorname{dist}\left(I_{0}, \mathbb{R} \backslash I\right)>\delta \text {. }
$$

Let $\mathcal{E}_{0}$ denote the eigenspace $\operatorname{span}\left\{e_{n}: \lambda_{n} \in I_{0}\right\} \in$ $L_{0}^{2}(\mathcal{D})$ associated with $I_{0}$ and $E_{0}$ the spectral projection onto $\mathcal{E}_{0}$. Let $\mathcal{E}_{1 / L}$ denote the subspace of the Hilbert space $\mathcal{H}:=L_{0}^{2}(\mathcal{D})$ invariant under the action of $\Xi_{L}$ and $E_{1 / L}$ the projection (not necessarily orthogonal) onto $\mathcal{E}_{1 / L}$.

The projection $E_{1 / L}$ is defined by the following set of equations:

$$
\left(I-E_{1 / L}\right) \Xi_{L}^{-1} E_{1 / L}=0
$$

(i.e. $\mathcal{E}_{1 / L}$ is an invariant subspace) and the two conditions

$$
E_{1 / L} E_{0}=E_{1 / L} \quad \text { and } \quad E_{0} E_{1 / L}=E_{0} .
$$

Note that $\Gamma$ under (V.2) $E_{1 / L}$ is a projector

$$
E_{1 / L}^{2}=\left(E_{1 / L} E_{0}\right) E_{1 / L}=E_{1 / L}\left(E_{0} E_{1 / L}\right)=E_{1 / L} E_{0}=E_{1 / L},
$$

which is consistent with $E_{0}$ in the sense that $\lim _{L \rightarrow \infty} E_{1 / L}=E_{0}$.

We shall prove that $\Gamma$ provided $V_{L}:=\Xi_{L}^{-1}-\partial^{-2}$ is bounded $\Gamma E_{1 / L}$ depends analytically on $1 / L$ and $\mathcal{E}_{1 / L}$ tends to $\mathcal{E}_{0}$ as $1 / L \rightarrow 0$. The proof of this statement uses equation (V.1) to write an integral equation. For simplicity $\Gamma$ we shall drop the index $L$ of the quantities
$\Xi_{L}^{-1} \Gamma V_{L} \Gamma \mathcal{E}_{1 / L}$ and $E_{1 / L}$

Our starting point begins with equation

$$
\begin{aligned}
(1-E) \partial^{-2} E & =\partial^{-2} E-E \partial^{-2} E \\
& =\partial^{-2} E-E \partial^{-2}
\end{aligned}
$$

which comes from the following facts. The operator $\partial^{-2}$ commutes with the spectral projector $E_{0}$. Using this and equations (V.2) Гwe have 


$$
E \partial^{-2} E=E E_{0} \partial^{-2} E=E \partial^{-2} E_{0} E=E \partial^{-2} E_{0}=E E_{0} \partial^{-2}=E \partial^{-2},
$$

and this implies the second line of (V.3). The commutation relation $\left[\partial^{-2}, E_{0}\right]=0$ allows us to replace $E$ in the equation (V.3) by $Q:=E-E_{0}$

$$
(1-E) \partial^{-2} E=\partial^{-2} Q-Q \partial^{-2} .
$$

Combining (V.1) with (V.4) and using $\Xi^{-1}=\partial^{-2}+$ $V$ T gives

$$
\begin{aligned}
\partial^{-2} Q & =Q \partial^{-2}-(I-E) V E \\
& =\partial^{-2} Q-\left(I-E_{0}-Q\right) V\left(E_{0}+Q\right) .
\end{aligned}
$$

Since the interval $I_{0}$ is isolate from the rest of the spectrum $\Gamma \partial^{-2}$ is an invertible bounded operator in the subspace $\left(I-E_{0}\right) \mathcal{H}$. We can solve the left hand side of (V.5) for $Q$ by defining

$$
X f:= \begin{cases}\partial^{2} f & \text { if } f \in\left(I-E_{0}\right) \mathcal{H} \\ 0 & \text { otherwise. }\end{cases}
$$

Note that $X \partial^{-2}=\partial^{-2} X=I-E_{0}$ and $\|X\|<\delta^{-1}$.

Equation (V.5) can thus be written as

$$
Q=g(Q)
$$

where

$$
g(Q)=X \partial^{-2} Q-X\left(I+E_{0}-Q\right) V\left(Q-E_{0}\right) .
$$

Proposition V.1 The sequence $Q_{n}, n=0,1, \ldots$, of projectors defined by

$$
Q_{n}=g\left(Q_{n-1}\right),
$$

with initial condition $Q_{0}=0$ satisfies the conditions (V.2) and converges, $Q=\lim _{n \rightarrow \infty} Q_{n}$, to the unique solution of equation (V.6).

Proof. We have $\left\|Q_{n}\right\| \leq q<1$ for all $n \in \mathbb{N}$ provided $q$ is chosen small enough and $L$ is taken so large that if $\|Q\| \leq q$ then

$$
\|g(Q)\| \leq\|X\|\left\|E_{0} \partial^{-2}\right\| q+\delta^{-1}(1+q)^{2}\left\|V_{L}\right\| \leq q .
$$

Note that the smallness of $g$ depends on the smallness of $V$. Since

$$
\|X\|\left\|E_{0} \partial^{-2}\right\| \leq \frac{\left|I_{0}\right|}{\delta+\left|I_{0}\right|}:=\alpha<1,
$$

equation (V.8) holds provided

$$
\|V\| \leq \frac{(1-\alpha) \delta}{(1+q)^{2}} q .
$$

Now $\Gamma$ for fixed value of $q$ Tit can be shown (see ref. [F] for details)

$$
\left|g(Q)-g\left(Q^{\prime}\right)\right| \leq \theta\left|Q-Q^{\prime}\right|
$$

also holds with $\theta<1$ and this implies Proposition V.1 by the Banach fixed point theorem.

We have proven the existence of a unique projector $E_{1 / L}$ such that $\left\|Q_{1 / L}\right\|=\left\|E_{1 / L}-E_{0}\right\| \leq q$. Since $q$ can be made arbitrarily small by taking $L$ sufficiently large so that (V.9) holds $\Gamma$ we have $\lim _{L \rightarrow \infty}\left\|E_{1 / L}-E_{0}\right\|=0$ and $\mathcal{E}_{1 / L} \longrightarrow \mathcal{E}_{0}$ as $L \rightarrow \infty$.

To complete the proof of Theorem III.1 $\Gamma$ we need to find an orthogonal projector $E^{L}$ onto $\mathcal{E}^{L} \equiv \mathcal{E}_{1 / L}$ in order to get (III.8). This is achieved by setting

$$
E^{L}=E_{1 / L}\left(E_{1 / L}^{\dagger} E_{1 / L}\right)^{-1} E_{1 / L}^{\dagger}
$$

and noting that the inverse operator $\left(E^{\dagger} E\right)^{-1}$ exist because $\|Q\| \leq q$ implies

$$
\left\|E_{0} f\right\| \leq\|E f\|+q\|f\|=\|E f\|+q\left\|E_{0} f\right\|,
$$

for any $f \in \mathcal{H}$ such that $E_{0} f=f$. As a consequence $\|E f\| \geq(1-q)\|f\|$ and

$$
\left\langle f, E^{\dagger} E f\right\rangle \geq(1-q)^{2}\|f\| .
$$

One can show $\Gamma$ in addition $\Gamma$ that $\left[\Xi^{-1}, E^{L}\right]=0$ for all $L$. Therefore $\Gamma$ for any $\lambda \in I_{0} \cap \sigma\left(\partial^{-2}\right) \Gamma$

$$
\begin{aligned}
\left\|E^{L}\left(\Xi^{-1}-\lambda I\right) E^{L}\right\| & =\left\|\left(\Xi^{-1}-\lambda I\right) E^{L}\right\| \\
& \leq\left\|\left(\partial^{-2}-\lambda I\right)\left(E_{0}+\left(E^{L}-E_{0}\right)\right)\right\|+\left\|V E^{L}\right\| \\
& \leq\left\|\left(\partial^{-2}-\lambda I\right)\right\|+\left\|\partial^{-2}\right\|\left\|E^{L}-E_{0}\right\|+\left\|V_{L}\right\| .
\end{aligned}
$$


Since the right hand side goes to zero as $L \rightarrow \infty$ this concludes the proof of Corollary III.2.

\section{Diffusion Coefficient}

This section is devoted to the proof of Theorem II.5. The diffusion coefficient will be estimated throughout an expansion for the expectation of the inverse matrix $\Gamma$ $\mathbb{E}\left(\Delta_{L, w}\right)^{-1} \Gamma$ with $w$ satisfying the macroscopic homogeneity condition (II.10). This is justified in ref. [AKS] in view of the fact that $\left(\Delta_{L, w}\right)^{-1} \Gamma$ when properly scaled $\Gamma$ converge to its expectation for almost all environment $w$. Thus $\Gamma$ the formula $\left(\mathbb{E}\left(\Delta_{L, w}\right)^{-1}\right)^{-1} \sim \Delta_{L, \kappa}$ is expect to hold in the limit as $L \rightarrow \infty$. We will see that very important cancellations take place by inverting the series expansion of $\mathbb{E}\left(\Delta_{L, w}\right)^{-1}$.

A simple algebraic manipulation shows

$$
\left(-\Delta_{L, \bar{w}}\right)^{1 / 2} \frac{1}{-\Delta_{L, w}}\left(-\Delta_{L, \bar{w}}\right)^{1 / 2}=\frac{1}{I-D_{L, w}},
$$

where

$$
D_{L, w}:=\left(-\Delta_{L, \bar{w}}\right)^{-1 / 2}\left(\Delta_{L, w}-\Delta_{L, \bar{w}}\right)\left(-\Delta_{L, \bar{w}}\right)^{-1 / 2}
$$

is a well defined matrix since $\Gamma$ in view of (II.2) and (II.10) $\Gamma-\Delta_{L, w}$ is positive and the square root of $-\Delta_{L, \bar{w}}$ can be taken.

Choosing $\bar{w}=\mathbb{E} w_{b}$ and use (II.1) to write $\Delta_{L, \bar{w}}=$ $\bar{w} \Delta_{L}$ where $\Delta_{L}$ is the finite difference Laplacian with 0 -Dirichlet boundary condition on $\Lambda$ Tequation (VI.2) can be written as

$$
D_{L, w}=\left(-\Delta_{L}\right)^{-1 / 2} \Delta_{L, \alpha}\left(-\Delta_{L}\right)^{-1 / 2},
$$

where $\alpha=\left\{\alpha_{b}\right\}$ given by $\alpha_{b}=w_{b} / \bar{w}-1 \Gamma$ are i.i.d. random variables with mean $\mathbb{E} \alpha_{b}=0 \Gamma$ such that

$$
\left|\alpha_{b}\right| \leq \delta<\frac{1}{2}
$$

holds in view of (II.10).

Equation (VI.1) suggests us the use of Neumann series to develop a formal expansion of $\left(\Delta_{L, w}\right)^{-1}$ in power of $D_{L, w}$ due to the small parameter $\delta$. The remaining of this section is devoted to the pointwise convergence of the matrix element of $\left[\mathbb{E}\left(I-D_{L, w}\right)^{-1}\right]^{-1}$.

Using (VI.3) Гwe have

$$
\frac{1}{I-D_{L, w}}=I+\sum_{n \geq 1}\left(-\Delta_{L}\right)^{-1 / 2} \Delta_{L, \alpha}\left[\left(-\Delta_{L}\right)^{-1} \Delta_{L, \alpha}\right]^{n-1}\left(-\Delta_{L}\right)^{-1 / 2}
$$

To write (VI.5) in a more convenient form $\Gamma$ let $\nabla_{L}: \mathbb{R}^{\Lambda} \longrightarrow \mathbb{R}^{\mathbb{B}_{L}}$ be the finite difference operator:

$$
\left(\nabla_{L} u\right)_{\langle x y\rangle}=-\left(\nabla_{L} u\right)_{\langle y x\rangle}=\sigma_{\langle x y\rangle}\left(u_{y}-u_{x}\right) .
$$

where the sign $\sigma_{\langle x y\rangle}=\sum_{i}\left(y_{i}-x_{i}\right)= \pm 1$ Taccording to whether $\langle x y\rangle$ is positively $(=1)$ or negatively $(=-1)$ oriented. $\nabla_{L}$ maps a 0 -form $u$ into a 1 -forms $\nabla_{L} u$. Let $\nabla_{L}^{*}: \mathbb{R}^{\mathbb{B}_{L}} \longrightarrow \mathbb{R}^{\Lambda}$ be its adjoint $\left(\omega, \nabla_{L} u\right)=\left(\nabla_{L}^{*} \omega, u\right) \Gamma$ i.e. the finite divergent operator which maps a 1 -form $\omega$ into a $0-$ form $\nabla_{L}^{*} \omega$ given by

$$
\left(\nabla_{L}^{*} \omega\right)_{x}=\sum_{y:|x-y|=1} \omega_{\langle y x\rangle},
$$

and let $M_{\alpha}: \mathbb{R}^{\mathbb{B}_{L}} \longrightarrow \mathbb{R}^{\mathbb{B}_{L}}$ be the multiplication operator by $\alpha:\left(M_{\alpha} \omega\right)_{b}:=\alpha_{b} \omega_{b}$.

With these notations $\Gamma$ we have

$$
\Delta_{L, \alpha}=\nabla_{L}^{*} M_{\alpha} \nabla_{L},
$$

and its bilinear form reads

$$
\left(u, \Delta_{L, \alpha} v\right)=\left(\nabla u, M_{\alpha} \nabla v\right)=\frac{1}{L^{d}} \sum_{b} \alpha_{b}(\nabla u)_{b}(\nabla v)_{b},
$$

recovering expression (II.2) for the quadratic form.

Define

$$
\Phi:=\nabla_{L}\left(-\Delta_{L}\right)^{-1} \nabla_{L}^{*},
$$

and note that $\Gamma$ since $\left(-\Delta_{L}\right)_{x, y}^{-1}$ is the Coulomb potential between two unit charges located at $x$ and $y \Gamma \Phi_{b, b^{\prime}}$ is the dipole interaction potential between two unit dipoles located at $b$ and $b^{\prime}$. Note that $\Phi$ maps 1 -form into $1-$ form.

In view of (VI.6) and (VI.7) Tequation (VI.5) can be rewritten as

$$
\frac{1}{I-D_{L, w}}=I+\sum_{n \geq 1} \sum_{\Gamma \in \mathbb{B}_{L}^{n}} \alpha_{\Gamma} W_{\Gamma}
$$


where $\Gamma$ for $\Gamma=\left(b_{1}, b_{2}, \ldots, b_{n}\right) \Gamma$

$$
\alpha_{\Gamma}=\prod_{k=1}^{n} \alpha_{b_{k}},
$$

and

$$
\left(v, W_{\Gamma} u\right)=\frac{1}{|\Lambda|} \vartheta_{b_{1}} \Phi_{b_{1}, b_{2}} \Phi_{b_{2}, b_{3}} \cdots \Phi_{b_{n-1}, b_{n}} \nu_{b_{n}},
$$

with $\vartheta$ and $\nu$ being 1 -forms given by $\nabla\left(-\Delta_{L}\right)^{-1 / 2} v$ and $\nabla\left(-\Delta_{L}\right)^{-1 / 2} u$ Trespectively.

Concern the convergence $\Gamma$ as $\Lambda \nearrow \mathbb{Z}^{d} \Gamma$ of a generic term of the expansion (VI.8) $\Gamma$ the following remark is now in order.
Remark VI.1 The asymptotic behavior of the dipole potential $\Phi_{b, b^{\prime}}$ for $L>>\operatorname{dist}\left(b, b^{\prime}\right)>>1$ can be estimated by its spectrum decomposition ${ }^{3}$,

$$
\Phi_{b, b^{\prime}}=\frac{1}{L^{d}} \sum_{n \in \Lambda^{*}} \tilde{\lambda}_{n}\left(\nabla e_{n}^{L}\right)_{b}\left(\nabla e_{n}^{L}\right)_{b^{\prime}}
$$

where

$$
\tilde{\lambda}_{n}^{-1}=4 \sum_{k=1}^{d} \sin ^{2} \frac{\pi}{4 L} n_{k}
$$

and $e_{n}^{L}$ as in (III.27). If we take $b=\left\langle x x^{(i)}\right\rangle$ and $b^{\prime}=\left\langle y y^{(j)}\right\rangle$, where $z^{(k)}$ is a nearest site of $z$ whose components are given by $z_{\ell}^{(k)}=z_{\ell}+\delta_{k, \ell}$, and make a change of variables, $\varphi_{i}=(\pi / 2 L) n_{i}, i=1, \ldots, d$, we have

$$
\lim _{L \rightarrow \infty} \Phi_{b, b^{\prime}}=\frac{1}{4 \pi^{d}} \int_{[0, \pi]^{d}} d^{d} \varphi\left(\sum_{k=1}^{d} \sin ^{2}\left(\varphi_{k} / 2\right)\right)^{-1} \nabla^{i} \nabla^{j} \prod_{k=1}^{d} \cos \left(\varphi_{k}\left(x_{k}-y_{k}\right)\right)
$$

where $\nabla^{k} f(z)=f\left(z^{(k)}\right)-f(z)$ is the difference operator in the $k$-th direction. The $|x-y|>>1$ behavior of
$\Phi_{b, b^{\prime}}$ is given by restricting the integral (VI.12) around a $\varepsilon$-neighborhood of 0 with $\varepsilon|x-y|=O(1)$ :

$$
\begin{aligned}
\Phi_{b, b^{\prime}} & \sim \frac{-1}{(2 \pi)^{d}} \int_{|\varphi| \leq \varepsilon} d^{d} \varphi \frac{\varphi_{i} \varphi_{j}}{\varphi^{2}} \tan \left(\varphi_{i}\left(x_{i}-y_{i}\right)\right) \tan \left(\varphi_{j}\left(x_{j}-y_{j}\right)\right) \prod_{k=1}^{d} \cos \left(\varphi_{k}\left(x_{k}-y_{k}\right)\right) \\
& \sim \frac{-1}{(2 \pi)^{d}|x-y|^{d}} \int_{|t| \leq O(1)} d^{d} t \frac{t_{i} t_{j}}{t^{2}} \\
& \sim \frac{1}{\left[\operatorname{dist}\left(b, b^{\prime}\right)\right]^{d}} .
\end{aligned}
$$

As a consequence $\Gamma \Phi_{b, b^{\prime}}$ is not summable in absolute value $\Gamma$

$$
\sum_{b^{\prime} \in \mathbb{B}_{L}}\left|\Phi_{b, b^{\prime}}\right| \sim \log L
$$

and the uniform convergence with respect to $\Lambda$ of the $\Gamma$-summation in (VI.8) requires cancellations due to the dipole orientations (see ref. [PPNM]).
We shall exhibit in the following another kind of cancellation due to the inversion of the expected value of (VI.8).

Inverting the expectation of (VI.8) gives

$$
\left[\mathbb{E}\left(I-D_{L, w}\right)^{-1}\right]^{-1}=I-\Theta_{L}
$$

where

\footnotetext{
${ }^{3}$ Since we have not rescaled the space $\Lambda \subset \mathbb{Z}^{d}$, it is convenient to introduce a base $\left\{\hat{e}_{n}^{L}\right\}_{n \in \Lambda^{*}}, \Lambda^{*}=\{1, \ldots, 2 L-1\}^{d}$, normalized with respect to the scalar product $((u, v)):=\sum_{x \in \Lambda} u_{x} v_{x}=L^{d}(u, v): \hat{e}_{n}^{L}=e_{n}^{L} / \sqrt{L^{d}}$. The spectrum resolution of the identity is written in terms of this base.
} 


$$
\Theta_{L}=\sum_{k \geq 1}(-1)^{k+1} \sum_{n_{1}, \ldots, n_{k}} \sum_{\Gamma=\left(\Gamma_{1}, \ldots, \Gamma_{k}\right)} \mathbb{E} \alpha_{\Gamma_{1}} \cdots \mathbb{E} \alpha_{\Gamma_{k}} W_{\Gamma}
$$

and $\Gamma \in \mathbb{B}_{L}^{n_{1}} \times \cdots \times \mathbb{B}_{L}^{n_{k}} \Gamma$ with $n_{i} \geq 1$. Note that $W_{\Gamma}=W_{\Gamma_{1}} \cdots W_{\Gamma_{k}}$.

To see how the log-divergent terms in (VI.14) cancel out $\Gamma$ it is convenient to use graph-theoretical language. A graph $G$ consists of two sets $(V, E): V=\left\{v_{1}, \ldots, v_{s}\right\}$ is the vertex set and $E=\left\{e_{1}, \ldots, e_{s^{\prime}}\right\}$ the connecting set of edges. To each edge $e$ its assigned an ordered pair of vertices $\left(v v^{\prime}\right)$ (its extremities) which are called adjacent if $v \neq v^{\prime}$; otherwise $e$ is said to be a "loop". To the problem at our hand $\Gamma$ we shall identify the bonds $\left\{b_{1}, \ldots, b_{n}\right\}$ as a the vertex set of a graph $G$ whose connectivity is determined by the presence of interactions $\Phi_{b, b^{\prime}}$.

Two graphs $G$ and $G^{\prime}$ are isomorphic (denoted $G \sim G^{\prime}$ ) if there is a one-to-one correspondence between their elements which preserves the incidence relation. A path $\Gamma$ on $G$ is an ordered sequence $\left\{v_{i_{0}}, e_{i_{1}}, \ldots, e_{i_{n}}, v_{i_{n}}\right\}$ of alternately vertices and edges of $G$ such that $e_{i_{k}}=\left(v_{i_{k-1}} v_{i_{k}}\right)$ holds for each $k$; the edges $\left\{e_{i_{1}}, \ldots, e_{i_{n}}\right\}$ are the steps of the path and the vertices $\left\{v_{i_{0}}, \ldots, v_{i_{n}}\right\}$ are the points visited by the path. $\Gamma$ may be identified with one of these ordered sets since it can be uniquely determined by each of them. Two vertices $v, v^{\prime} \in V$ may be connected by more than one path. A graph $G$ is said to be connected if any two vertices $v, v^{\prime}$ can be joined by at least one path $\Gamma$ on $G$. The components of a non-connected graph are its maximum connected subgraphs. Given two vertices $v, v^{\prime} \Gamma$ the disconnecting set of edges is a set whose removal from the graph $G$ destroys all paths between $v, v^{\prime}$. A cut-set is a minimal set of edges the removal of which from a connected graph $G$ causes it to fall into two components $G_{1}, G_{2}$.

Turning back to equation (VI.14) Гone may interpret $\Gamma=\left\{b_{1}, \ldots, b_{n}\right\}$ as a set of vertices visited by a path. In view of the fact that $\alpha_{b}$ has zero mean $\Gamma$ we have

$$
\mathbb{E} \alpha_{\Gamma} \equiv \bar{\alpha}_{\Gamma}=0
$$

if there exist at least one bond $b_{i}$ which are not repeated in the list $\Gamma=\left\{b_{1}, \ldots, b_{n}\right\}$.
The condition (VI.16) says that the path $\Gamma$ must visit each vertex at least twice otherwise its contribution to (VI.14) vanishes. The set of distinct bonds $V=\left\{b_{i_{1}}, \ldots b_{i_{s}}\right\}$ and edges $E=\left\{\left(b_{1} b_{2}\right), \ldots,\left(b_{n-1} b_{n}\right)\right\}$ form a connected graph $G$ with even valency $\mathcal{V}(b) \geq 4$ for each vertex $b \in G$. Graphs with this property will be called admissible graphs. Note that each path $\Gamma$ yields only one graph $G$ but there are possibly many $n$-step paths covering each edge $\left(b_{i-1} b_{i}\right)$ of $G$ exactly once which starts at $b_{1}$ and ends at $b_{n}$. If we denote by $[\Gamma]_{G}$ the set of all paths $\Gamma$ satisfying these conditions for a given admissible graph $G \Gamma$ we have

Proposition VI.2 Equation (VI.15) can be written as

$$
\Theta_{L}=\sum_{n \geq 1} \sum_{\substack{G:|E|=n-1 \\ \text { admissible }}} A_{G} W_{G}
$$

with

$$
A_{G}:=\sum_{\Gamma \in[\Gamma]_{G}} \sum_{\Gamma=\left(\Gamma_{1}, \ldots, \Gamma_{s}\right)}(-1)^{s+1} \bar{\alpha}_{\Gamma_{1}} \cdots \bar{\alpha}_{\Gamma_{s}},
$$

where we sum over all sizes $n \in \mathbb{N}, n \geq 1$, all admissible graphs $G$ of size $|E|=n$, over all paths $\Gamma$ in $[\Gamma]_{G}$ and over all decompositions of $\Gamma$ into $s, s \geq 1$, successive paths $\left(\Gamma_{1}, \ldots, \Gamma_{s}\right)$, each of which capable of generating admissible graphs $G_{i}$. Here, with the notation of (VI.10) and footnote in Remark VI.1,

$$
\left(\left(v, W_{G} u\right)\right)=\vartheta_{b_{1}}\left(\prod_{\left\langle b b^{\prime}\right\rangle \in E(G)} \Phi_{b, b^{\prime}}\right) \nu_{b_{n}},
$$

for $n>1$ with $\Phi_{b_{1}, b_{1}}=1$ for $n=1$ (the case that $G$ is the trivial graph $\left.\left(\left\{b_{1}\right\}, \emptyset\right)\right)$.

We shall in the sequel state two lemmas and prove Theorem II.5 under an extra assumption.

Lemma VI.3 If $G$ is an admissible graph with at least one cut-set contained one edge (i.e. $G$ falls into two components by cutting a single edge), then $A_{G}=0$. 
Lemma VI.4 There exist a constant $C_{[G]}<\infty$, depending on the equivalence class $[G]$ of isomorphic graphs $G$, such that

$$
\sum_{\substack{G^{\prime}: G^{\prime} \sim G \\ b_{1}, b_{n} \text { fixed }}}\left|\prod_{\left\langle b b^{\prime}\right\rangle \in E\left(G^{\prime}\right)} \Phi_{b, b^{\prime}}\right| \leq \frac{C_{[G]}}{\left[1+\operatorname{dist}\left(b_{1}, b_{n}\right)\right]^{2 d}}
$$

holds uniformly in $L$ for all admissible graph $G$ with $|E|=n$ and cut-sets with no less than two elements.

Remark VI.5 The proof of Lemmas VI.3 and VI.4 are essentially given in [AKS] (see Assertions $I$ and II of Section 4). Note that our estimate (VI.20) have not included the logarithmic corrections which appears in that reference. To get rid of these one has to control the loop subgraphs of $G$ carefully as it is done in the ref. [PPNM]. The uniform upper bound (VI.20) results from the hypothesis that $G$ remains connected by cutting one single edge.

Graphs with single edge cut-sets do not contribute to (VI.17) due to the following cancellation in Lemma VI.3.

Proof of Lemma VI.3. Let $\left(b_{i} b_{i+1}\right)$ be the only edge of a cut-set and let $\Gamma=\left(\Gamma_{1}, \ldots, \Gamma_{s}\right)$ be a decomposition of a path in $G$. Either both $b_{i}$ and $b_{i+1}$ belongs to some $\Gamma_{j}$ or they belong to two successive ones. We call the latter decomposition type $A$ and the former type $B$. It turns out that there is an one-to-one correspondence between type $A$ and type $B$ decompositions differing only by the splitting of $\Gamma_{j}$ into two elements $\Gamma_{j}^{(1)}$ and $\Gamma_{j}^{(2)}$. Lemma VI.3 follows from the fact that the contribution to (VI.18) of a pair of decompositions established by this correspondence have the same absolute value with opposite signals. Note $\bar{\alpha}_{\Gamma_{j}}=\bar{\alpha}_{\Gamma_{j}^{(1)}} \bar{\alpha}_{\Gamma_{j}^{(2)}}$ if the edge $\left(b_{i} b_{i+1}\right)$ bridges the two paths $\Gamma_{j}^{(1)}$ and $\Gamma_{j}^{(2)}$.

In view of Proposition VI.2 and Lemmas VI.3 and VI.4Tequation (VI.15) can be estimated as

$$
\left|\left(\left(v, \Theta_{L} u\right)\right)\right| \leq \sum_{b, b^{\prime} \in \mathbb{B}_{L}} \vartheta_{b} \frac{K_{L}}{\left[1+\operatorname{dist}\left(b, b^{\prime}\right)\right]^{2 d}} \nu_{b^{\prime}}
$$

where

$$
K_{L}=\sum_{n \geq 1} \sum_{\substack{[G]:|\in|=n-1 \\ \text { admissible }}} A_{[G]} C_{[G]}
$$

with the sum running over the equivalence classes $[G]$ of isomorphic admissible graphs $G$ of size $|E|=n$ and $C_{[G]}$ as in Lemma VI.4. Note that $A_{G}=A_{[G]}$.
Now we show that $\Gamma$ if one uses $\Gamma$ as in refs. [AKS] and [PPNM] The upper bound

$$
C_{[G]} \leq C^{r}
$$

for some geometric constant $C<\infty$ where $r=|V|$ is the number of vertices in $G \Gamma$ the equation (VI.22) cannot be bounded uniformly in $L$. Taking into account property (VI.4) $\Gamma$

$$
\left|\bar{\alpha}_{\Gamma_{1}} \cdots \bar{\alpha}_{\Gamma_{s}}\right| \leq \delta^{n}
$$

holds uniformly in $\Gamma$ and equation (VI.22) can be bounded by

$$
K_{L} \leq \sum_{n \geq 1}\left(\begin{array}{c}
2 n-1 \\
n
\end{array}\right) \delta^{n} \sum_{r=1}^{\min (n,|\Lambda|)} \Pi(n, r) C^{r}
$$

Here $\Gamma$ we have identified each path $\Gamma=\left\{b_{1}, \ldots, b_{n}\right\}$ in a given graph $G=(V, E)$ of size $|E|=n-1$ with a partition $P=\left(P_{1}, \ldots, P_{r}\right)$ of $\{1,2, \ldots, n\}$ into $r=|V|$ pairwise disjoint subsets. This association is one-to-one since $\Gamma$ is an ordered set of elements. Note that each component $P_{j}$ corresponds to one vertex $b_{i_{1}}=b_{i_{2}}=\cdots=b_{i_{p}}$ of $G$ visited $p=\left|P_{j}\right|$ times by the path $\Gamma$. One can thus replace the sum over all equivalent classes of graphs $[G]$ and over all paths $\Gamma$ in $[G]$ by the sum over all partitions $P$. The factor $\Pi(n, r)$ counts the number of partitions of $\{1,2, \ldots, n\}$ into $r$ subsets. The bound (VI.25) disregards the fact that $G$ is an admissible graph. Also $\Gamma$ the consistency of each decomposition $\Gamma=\left(\Gamma_{1}, \ldots, \Gamma_{s}\right)$ into paths $\Gamma_{i}$ 's which gives rise to admissible graphs has been not considered. The binomial factor $\left(\begin{array}{c}2 n-1 \\ n\end{array}\right) \leq 4^{n}$ counts the decomposition of $\Gamma$ with $n$ steps into any number of paths with the number of steps $\leq n$ (the cardinality of the set $\left.\left\{1 \leq i_{1} \leq i_{2} \leq \cdots \leq i_{n-1} \leq n-1\right\}\right)$. In addition $\Gamma$ for the upper limit in the second sum we note that $r=|V| \leq(2 L-1)^{d}$ (the number of vertices of $G$ cannot be larger than the number of sites in $\Lambda$ ).

Equation (VI.25) cannot be uniformly bounded since $\Gamma$ from the recursion relation $\Pi(n, r)=\Pi(n-1, r-$ $1)+r \Pi(n-1, r)($ see $[\mathrm{W}]) \Gamma$ we have

$$
\Pi(n, r) \geq r \Pi(n-1, r) \geq r^{n-r} \Pi(r, r)=r^{n-r}
$$

which gives a factorial growth 


$$
\sum_{r=1}^{\min (n,|\Lambda|)} \Pi(n, r) C^{r} \geq\left\{\begin{array}{lll}
(C n / 2)^{n / 2} & \text { if } & n \leq|\Lambda| / 2 \\
(C|\Lambda| / 2)^{n-|\Lambda| / 2} & \text { if } & n>|\Lambda| / 2
\end{array}\right.
$$

after replacing the sum by the term with $r=$ $\min (n,|\Lambda|) / 2$.

A sharper upper bound for (VI.23) may be assumed if one think of $C_{[G]}$ as being given by

$$
C_{[G]}=\sup _{G^{\prime} \sim G}\left|\prod_{\left\langle b b^{\prime}\right\rangle \in E\left(G^{\prime}\right)} \Phi_{b, b^{\prime}}\right|\left(1+\operatorname{dist}\left(b_{1}, b_{n}\right)\right)^{2 d} .
$$

As one varies the partition $P$ of $\{1, \ldots, n\} \Gamma$ the graph $G \Gamma$ and the path $\Gamma$ over it $\Gamma$ varies accordingly and the decay of $\Phi_{b, b^{\prime}}$ in this formula can be useful. We propose that $C_{[G]}=C_{n, r}$ depends on the number of vertices $r=|V|$ and edges $n=|E|$ as follows.

Conjecture VI.6 Let $\widetilde{\Pi}(n, r)=C_{n, r} \Pi(n, r)$. There exist a geometric constant $C<\infty$ such that

$$
\widetilde{\Pi}(n, r) \leq \widetilde{\Pi}(n-1, r-1)+C \widetilde{\Pi}(n-1, r)
$$

holds for $n, r \in \mathbb{N}, n \geq r$ with $\widetilde{\Pi}(r, r)=C^{r}$.

Note that $\Pi(n, r)$ satisfies (VI.27) with $C$ replaced by $r$. Assuming (VI.27) and using that $\widetilde{\Pi}(1,1)=C$ and $\widetilde{\Pi}(k, l)=0$ if $k<l \Gamma$ we have

$$
\begin{aligned}
\sum_{r=1}^{n} \widetilde{\Pi}(n, r) & \leq \sum_{r=2}^{n} \widetilde{\Pi}(n-1, r-1)+C \sum_{r=1}^{n-1} \widetilde{\Pi}(n-1, r) \\
& =(1+C) \sum_{r=1}^{n-1} \widetilde{\Pi}(n-1, r) \\
& \leq C(1+C)^{n}
\end{aligned}
$$

which leads (VI.25) to be bounded by

$$
K_{L} \leq \sum_{n \geq 1}(4 \delta)^{n} \sum_{r=1}^{\min (n,|\Lambda|)} \widetilde{\Pi}(n, r) \leq \frac{C \delta^{\prime}}{1-\delta^{\prime}}
$$

where $\delta^{\prime}=4(1+C) \delta$.

This concludes the preliminaries and we are now ready to prove Theorem II.5. We observe at this point that no restrictions about the random variables $\alpha_{b}$ 's has been made beside (VI.16) and (VI.24) with $\delta$ small enough. Has Conjecture VI.6 been proved one could work along similar expansions to show that $\left(-\Delta_{L, \bar{w}}\right)^{-1 / 2}\left(-\Delta_{L, w}\right)\left(-\Delta_{L, \bar{w}}\right)^{-1 / 2}$ converges to $\left[\mathbb{E}\left(I-D_{L, w}\right)^{-1}\right]^{-1}$ with probability 1 .

Proof of the upper bound of II.11. Let us recall some facts about the matrix $-\Delta_{L, w}$. By equation (II.2) it is a positive definite matrix and its square root is well defined. We also have $\mathbb{E}\left(-\Delta_{L, w}\right)=-\Delta_{L, \bar{w}}=$ $-\bar{w} \Delta_{L}$ and $\Gamma$ by Lemma III.1 $i\left(-\Delta_{L, w}\right)^{-1} i^{\dagger} / L^{2}$ converges with probability 1 to $\left(-\partial^{2}(\kappa)\right)^{-1}$ exactly as $i\left(-\nabla_{L} \cdot \kappa \nabla_{L}\right)^{-1} i^{\dagger} / L^{2}$ does.

In view of this $\Gamma$ we can apply Schwarz inequality to the following identity ${ }^{4}$ :

$$
I=\left(\mathbb{E}\left(-\Delta_{L, w}\right)^{1 / 2}\left(-\Delta_{L, w}\right)^{-1 / 2}\right)^{2} \leq \mathbb{E}\left(-\Delta_{L, w}\right) \mathbb{E}\left(-\Delta_{L, w}\right)^{-1}
$$

\footnotetext{
${ }^{4}$ In the following, for any two matrices $A$ and $B, A \leq B$ means $(u, A u) \leq(u, B u)$ for all vectors $u$.
} 
in order to get

$$
\left(\mathbb{E}\left(-\Delta_{L, w}\right)^{-1}\right)^{-1} \leq-\bar{w} \Delta_{L}
$$

which implies $\kappa \leq \bar{w}$ and concludes our assertion.

Proof of the lower bound of II.11. From equations

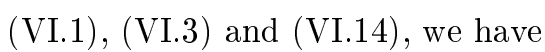

$$
\begin{aligned}
\left(\mathbb{E}\left(-\Delta_{L, w}\right)^{-1}\right)^{-1} & =-\bar{w} \Delta_{L}-\bar{w}\left(-\Delta_{L}\right)^{1 / 2} \Theta_{L}\left(-\Delta_{L}\right)^{1 / 2} \\
& =-\bar{w} \nabla^{*} R_{L} \nabla
\end{aligned}
$$

where $R_{L}: \mathbb{R}^{\mathbb{B}_{L}} \longrightarrow \mathbb{R}^{\mathbb{B}_{L}}$ is a matrix whose elements $\Gamma$ in view of (VI.21) and (VI.29) Tare bounded by

$$
\left(R_{L}\right)_{b, b^{\prime}} \geq \delta_{b, b^{\prime}}-\frac{K_{L}}{\left[1+\operatorname{dist}\left(b, b^{\prime}\right)\right]^{2 d}}
$$

with $K_{L} \leq \delta^{\prime} C /\left(1-\delta^{\prime}\right)$. Note from equations (VI.30) and (VI.31) that $\Theta_{L}$ is a positive matrix.

Using the isometry operators (III.1) and (III.2) and the fact that $i^{\dagger} i$ is the identity matrix in $\mathbb{R}^{|\Lambda|} \Gamma$ we have $L^{2} i\left(\mathbb{E}\left(-\Delta_{L, w}\right)^{-1}\right)^{-1} i^{\dagger}=$ $\bar{w}\left(L i \nabla^{*} i^{\dagger}\right)\left(i R_{L} i^{\dagger}\right)\left(L i \nabla i^{\dagger}\right) \quad$ with $L i \nabla^{*} i^{\dagger}$ and $L i \nabla i^{\dagger}$ converging in $L_{0}^{2}(\mathcal{D})$ to the operator $\partial=$ $\left(\partial / \partial \xi_{1}, \ldots, \partial / \partial \xi_{d}\right)$. In addition $\Gamma$ we claim that the kernel of $i\left(I-R_{L}\right) i^{\dagger}$ converges in distribution $\Gamma$ as $L \rightarrow \infty \Gamma$ to the delta function $\delta(\eta, \xi)$ times a $d \times d$ matrix $\varrho \Gamma$ since the matrix elements of $R_{L}$ decay faster than $1 / \operatorname{dist}\left(b, b^{\prime}\right)^{d}$ as $\operatorname{dist}\left(b, b^{\prime}\right) \rightarrow \infty$. For this $\Gamma$ note that $i\left(I-R_{L}\right) i^{\dagger}(\eta, \xi)=\mathcal{O}\left(L^{-d}\right)$ if $\eta \neq \xi$ and $=\mathcal{O}\left(L^{d}\right)$ if $\eta=\xi$.

Whether $\varrho$ is a diagonal matrix cannot be decided by our estimates. The results from this section leads to $\left(i R_{L} i^{\dagger}\right)_{i, j}(\eta, \xi) \longrightarrow\left(\delta_{i, j}+\varrho_{i, j}\right) \delta(\eta, \xi)$ and this implies

$$
\kappa=\bar{w}(1-\varrho)
$$

where 1 is the $d \times d$ identity and $\varrho$ is a positive matrix satisfying $\varrho \leq \delta^{\prime} C /\left(1-\delta^{\prime}\right)$.

\section{Acknowledgment}

We wish to thank Luiz R. Fontes for helpful discussions. R. da Silva was supported by $\mathrm{CNPq}$ under the PIBIC project and D.H.U. Marchetti was partially supported by FAPESP and CNPq.

\section{References}

[ABO] S. Alexander J. Bernasconi and R. Orbach. "Spectral diffusion in a one-dimensional percolation model", Phys. Rev. B 17, 4311-4314 (1978)

[ABSO] S. Alexander, J. Bernasconi, W. R. Schneider and R. Orbach. "Excitation dynamics in random onedimensional systems", Rev. Mod. Phys. 53, 175198 (1981)

[AKS] V. V. Anshelevich, K. M. Khanin and Ya. G. Sinai. "Symmetric random walks in random environments", Commun. Math. Phys. 85, 449-470 (1982)

[AV] V. V. Anshelevich and A. V. Vologodskii. "Laplace operator and random walk on one-dimensional nonhomogeneous lattice", Journ. Stat Phys. 25, 419-430 (1981)

[BSW] J. Bernasconi, W. R. Schneider and W. Wyss. "Diffusion and hopping conductivity in disordered onedimensional lattice systems", Z. Physik B 37, 175184 (1980)

[D] F. J. Dyson. "The dynamics of a disordered chain of harmonic oscilators", Phys. Rev. 92, 1331-1338 (1953)

[F] K. O. Friedrichs. "Perturbation of spectra in Hilbert space". AMS Lect. in Appl. Math., Providence, Rhode Island (1965)

[FIN] L. R. G. Fontes, M. Isopi and C. M. Newman. "Chaotic time dependence in a disordered spin system", Preprint (1998)

[J] Alouf Jirari. "Second-order Sturm-Liouville difference equations and orthogonal polynomials" Memoirs of AMS 542 (1995)

[K] P. W. Kasteleyn. "Graph theory and crystal physics", in Graph theory and theoretical physics, ed. F. Harary, AP New York (1967)

[KKS] H. Kesten, M. W. Koslow and F. Spitzer. "A limit law for random walk in random environment", Compos. Math. 30, 145-168 (1975)

[Ku] Rolf Künnemann. "The diffusion limit for reversible jump processes on $\mathbb{Z}^{d}$ with ergodic random bond conductivities", Commun. Math. Phys. 90, 27-68 (1983) 
[LM] Elliot H. Lieb and Daniel C. Mattis. "Mathematical physics in one-dimension: Exactly soluble models of interacting particles", Academic Press (1966)

[MFGW] A. de Masi, P. A. Ferrari, S. Goldstein an W. D. Wick. "An invariant principle for reversible Markov processes. Aplications to random motions in random environments", Journ. Stat. Phys. 55, 787-855 (1989)

[PPNM] Aldo Procacci, Emmanuel Pereira, Armando G. M. Neves and Domingos H. U. Marchetti. "Coulomb interaction symmetries and the Mayer series in two dimensional dipole gas", Journ. Stat. Phys. 87, 877-889 (1997)
[PV] G. Papanicolaou and S. R. S. Varadhan. "Diffusion with random coefficients", in Statistics and Probability: Essays in Honor of C. R. Rao", 547-552, North-Holland (1982)

[S] Barry Simon, "Functional integration and quantum physics", Academic Press (1979)

[Si] Ya. G. Sinai. "The limiting behavior of a onedimensional random walk in a random medium", Theo. of Prob. and its Appl. 27, 256-268 (1982)

[So] F. Solomon. "Random walks in a random environment", Ann. Prob. 3, 1-31 (1975)

[W] Herbert S. Wilf. "Generatingfunctionology", second edition, Academic Press (1994) 